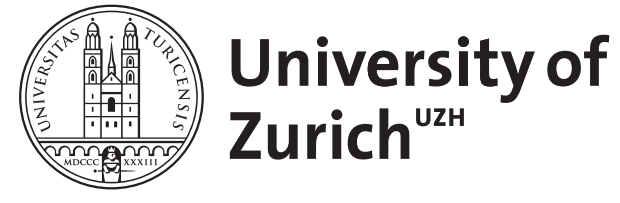
Archive

University of Zurich

University Library

Strickhofstrasse 39

CH-8057 Zurich

www.zora.uzh.ch

Year: 2010

\title{
Perfektionismus und Pathologien der Selbstverwirklichung
}

Schefczyk, Michael

DOI: https://doi.org/10.1524/dzph.2010.0057

Posted at the Zurich Open Repository and Archive, University of Zurich

ZORA URL: https://doi.org/10.5167/uzh-96903

Journal Article

Originally published at:

Schefczyk, Michael (2010). Perfektionismus und Pathologien der Selbstverwirklichung. Deutsche Zeitschrift für Philosophie, 58(5):741-757.

DOI: https://doi.org/10.1524/dzph.2010.0057 


\title{
Perfektionismus und Pathologien der Selbstverwirklichung
}

\author{
Von MICHAEL SCHEFCZYK (Lüneburg)
}

\begin{abstract}
Selbstverwirklichung ist ein schillerndes Wort. Auf der einen Seite bezieht es sich auf den Wunsch nach einem Leben, in dem man selber mehr eine als nur dienende Rolle spielt. Es lässt sich in dieser Bedeutung Konzepten wie „Fremdbestimmung“, „Ausbeutung“ und „Instrumentalisierung“ entgegensetzen: Menschen arbeiten an ihrer Selbstverwirklichung, wenn und soweit es ihnen möglich ist, selbstbestimmt zu handeln. So verstanden, bezeichnet das Wort etwas, dessen Berechtigung wohl nur wenige bestreiten wollen. ${ }^{1}$

Auf der anderen Seite gilt der Anspruch auf Selbstverwirklichung vielen als symptomatisch für eine Geisteshaltung, die mit nichts anderem als der selbstsüchtigen Erschließung eigener Lust- und Erfahrungsquellen beschäftigt ist und die Verantwortung für andere und anderes ablehnt. Die Zurückweisung einer rein dienenden und fremdbestimmten Rolle wird in das Extrem einer Existenz getrieben, die sich nur noch um sich selber zu kümmern bereit ist. „Selbstverwirklichung“ gilt dann als Synonym unmoralischer und möglicherweise auch unglücksträchtiger „Egozentrik“.
\end{abstract}

\section{Perfektionismus - einige Unterscheidungen}

Der schillernde Charakter der Selbstverwirklichung ist wohl in den meisten philosophischen Analysen des Begriffs thematisiert worden. Wahre und echte Formen werden ,pathologischen Ausprägungen“" entgegengesetzt. Die bereits angesprochene Kritik an einem egozentrisch verkürzten Verständnis dürfte die wohl häufigste Degenerationsdiagnose sein. In diesem Aufsatz beschäftigt mich die Frage, wie das Verhältnis von Selbstverwirklichung und Moral bestimmt werden kann, und ich werde zu diesem Zweck zwei Grundmodelle unterscheiden. Das eine, in vielfältigen Varianten auftretende Modell nimmt an, dass sich Menschen in einem uneingeschränkten Sinne selbst verwirklichen können, ohne sich um Fragen moralischer Richtigkeit zu kümmern. Ich nenne dies das Unabhängigkeitsmodell. Ein ganz und gar nicht-moralisches Leben steht, dem Unabhängigkeitsmodell zufolge, der Selbstverwirklichung nicht entgegen. Was ein nicht-moralisches Leben genau sein soll, wäre natürlich im Einzelnen zu klären, aber

1 „Die gewöhnliche Bedeutung von Selbstverwirklichung im modernen Sprachgebrauch ist etwa die folgende: Ein Individuum findet heraus, woran ihm besonders gelegen ist, was ihm das Leben lebenswert macht, was für ein Mensch sie oder er sein möchte, welche Fähigkeiten es hat, welche Tätigkeiten, welcher Genuss und welche ,Bezugspersonen“ sein Leben erfüllen könnten.“ (Siep 2002, 41) 
für den Moment reicht ein grobkörniges Verständnis aus. Das Unabhängigkeitsmodell findet sich in je unterschiedlicher Ausprägung beispielsweise bei Thomas Carlyle, Friedrich Nietzsche, in der existenzphilosophischen Tradition, aber auch in zeitgenössischen Formen des Perfektionismus, namentlich bei Thomas Hurka. ${ }^{2}$ Es kann in zwei Unterformen auftreten, die ich Vereinbarkeits- respektive Unvereinbarkeitsmodell taufen möchte. Vereinbarkeitsmodelle nehmen an, Selbstverwirklichung könne sowohl eine moralische als auch eine nicht-moralische Form annehmen, während Unvereinbarkeitsmodelle meinen, nur ein nicht-moralisches Leben könne wahrhaft zur Selbstverwirklichung führen.

Das vom Unabhängigkeitsmodell zu unterscheidende Abhängigkeitsmodell dürfte häufiger vertreten worden sein und vertreten werden als jenes und lehrt, dass eine Orientierung an Fragen moralischer Richtigkeit für die Selbstverwirklichung notwendig ist. Eine Person, die ein nicht-moralisches Leben führt, verwirklicht sich nicht selbst. Die Rede von „Pathologien der Selbstverwirklichung“ ist vor allem in Abhängigkeitsmodellen zuhause und dient vornehmlich dem Zweck, nicht-moralische Lebensformen als - entgegen dem Anschein - unvereinbar mit Selbstverwirklichung zu kennzeichnen. Dabei gibt es natürlich, wie gesagt, eine große Bandbreite, „Moral“ und „Selbstverwirklichung“ zu bestimmen. ${ }^{3}$

Vor dem Hintergrund dieser typologischen Orientierungshilfen möchte ich den Fokus meiner Überlegungen im Folgenden weiter eingrenzen und untersuchen, wie das Verhältnis von Selbstverwirklichung und Moral in den perfektionistischen Konzepten Hegels und T. H. Greens gefasst wird. Oben hatte ich bereits angedeutet, dass keineswegs alle Perfektionistinnen die Auffassung vertreten, eine nicht-moralische Existenzform stelle eine pathologische Weise von Selbstverwirklichung dar. Dies mag für diejenigen verwirrend klingen, die davon ausgehen, dass perfektionistische Ansätze in erster Linie dadurch motiviert sind, die Idee der Selbstverwirklichung zu moralisieren. John Christman definiert den Perfektionismus beispielsweise als die Ansicht, dass Werte und moralische Prinzipien für eine Person unabhängig von ihrem eigenen Urteil (hinsichtlich dieser Werte und Prinzipien) Verbindlichkeit haben können. Diese Definition bündelt zwei Bedingungen: zum einen, dass Perfektionisten - Christman zufolge - Objektivistinnen mit Blick auf Werte, Lebensideale und moralische Prinzipien sind (a); zum anderen, dass sie Zwangsausübung für legitim halten, um den objektiven Werten, Prinzipien und Idealen im Leben der Individuen Geltung zu verschaffen

2 „Many perfectionists allow their views about human nature to be shaped by moral considerations and, as a result, make claims about that nature that are false. This is especially true of perfectionists whose theories are moralistic. [...] Moralism makes goodness by perfectionist standards in part the same as goodness by the lights of commonsense morality. It makes the degree to which humans develop their natures depend on the degree to which they fulfill popular notions of morality. Moralism is present in the perfectionism of Aristotle, Aquinas, and Green, and it dominates that of Kant. In my view it is a fundamental error. Humans are by no means necessarily virtuous [...]. Because moralism is such an error, the best perfectionism must be free of it. It must never characterize the good by reference to conventional moral rules, but always non-morally [...].“ (Hurka 1993, 19, 20) Und: „In defining human nature, we rejected moralism, the view that developing one's own nature requires other-regarding virtues. Although humans are essentially rational, their rationality can be realized as much in skilful burglary as in philanthropy.“ (Ebd., 62)

3 Eine weitere Modellvariante, die hier nicht weiter beachtet werden soll, bietet die Existenzphilosophie an, wenn sie die nicht-moralisch verstandene Selbstverwirklichung selbst zu einem moralischen Imperativ erklärt. Mir scheint, dass Michael Theunissen sie völlig zu Recht als moralphilosophisch unangemessen zurückweist: ,[...] Eine Philosophie vom Typ der Existenzphilosophie, die das sprachlich ganz richtig als vereinzelnde Individualisierung aufgefasste Selbstwerden zum ethischen Postulat erhebt, [ist] eine schlechte, unverbindliche Ethik." (Theunissen 1982, 13-14) 
(b). ${ }^{4}$ Was jedoch eine objektivistische Rechtfertigung staatlichen Zwangs erst perfektionistisch macht, das ist die darüber hinausgehende These, dass die betreffende Person ein besseres Leben hätte (dass sie ihr ,wahres Selbst“ in vollerem Umfang verwirklichen würde), wenn sie sich den besagten Werten und Prinzipien - auch entgegen ihrem eigenen Urteil - unterwerfen würde. Eine Pointe des politikphilosophischen Perfektionismus kann man gerade darin sehen, objektivistische Wert- und Moraltheorien mit der Legitimation staatlicher Macht auf der Basis individueller Autonomie versöhnen zu wollen. Die Unterwerfung der Individuen unter staatlichen Zwang geschieht im Namen ihres ,wahren Selbst“.

Unbestreitbar hat dieses Motiv in vielen Varianten des Perfektionismus, insbesondere in der hegelianischen Traditionslinie, eine herausragende Rolle gespielt. Doch wäre der Perfektionismus zu eng gefasst, wollte man ihn auf diese Funktion reduzieren. Zum einen sind Formen des Perfektionismus denkbar und auch tatsächlich formuliert worden, die die Existenz und Gültigkeit objektiver Werte bejahen, aber bestreiten, dass deren Erzwingung als legitime Realisierung des „wahren Selbst“ betrachtet werden könnte. In diese Richtung tendieren beispielsweise Liberale, die meinen, ein autonomes Leben sei objektiv gesehen (unabhängig von den Überzeugungen und Einstellungen der betroffenen Person) ein vollkommeneres Leben als ein nicht-autonomes; die aber nicht glauben, dass Individuen zur Verwirklichung dieses Wertes gezwungen werden könnten oder sollten. ${ }^{5}$ Mit anderen Worten, die Überzeugung, dass es objektive und für alle Handelnden verbindliche Werte und Prinzipien gebe, legt eine Person nicht darauf fest, die Ausübung staatlichen Zwangs zur Durchsetzung dieser Werte und Prinzipien für legitim oder geboten zu halten.

Noch in anderer Hinsicht ist Christmans These zu eng, dass den Kern des Perfektionismus die Auffassung ausmache, es gebe objektiv gültige Werte, die staatliche Zwangsausübung legitimierten. Der Perfektionismus ist nämlich nicht nur und vielleicht noch nicht einmal vorrangig eine politikphilosophische Theoriebildung. In seiner ethischen Ausformung widmet er sich der Frage, was ein Leben gut mache. Wie das obige Beispiel der Liberalen zeigt, die ein autonomes Leben für vollkommener halten als ein nicht-autonomes, Versuche ihrer staatlichen Erzwingung jedoch zurückweisen, lassen sich Formen des ethischen Perfektionismus mit solchen des politikphilosophischen Anti-Perfektionismus kombinieren. Umgekehrt ist aber der politikphilosophische Perfektionismus auf eine Form des ethischen Perfektionismus angewiesen, beruht er doch auf der Idee, dass die Verwirklichung bestimmter objektiver Werte jemandes Leben besser mache. Insofern gilt, dass der politikphilosophische Perfektionismus in der Regel einen ethischen Perfektionismus voraussetzt, aber nicht jeder ethische Perfektionismus einen politikphilosophischen Perfektionismus stützt. Neben dem Problem der legitimen Ausübung staatlicher Gewalt und des gelingenden Lebens stellt sich aber noch die Frage, welchen moralischen Pflichten Individuen unterliegen. So ist es sinnvoll, außer dem ethischen und dem politikphilosophischen auch noch einen moralphilosophischen Perfektionismus einzuführen. Moralphilosophische Perfektionistinnen sind überzeugt, dass Menschen moralisch verpflichtet sind, an ihrer Selbstverbesserung zu arbeiten.

Es fragt sich nun, wie sich der Perfektionismusbegriff allgemein und abstrakt genug fassen lässt, um dieser Vielfalt von Verwendungsweisen gerecht zu werden. Mit Blick auf die reiche Begriffsgeschichte scheint mir der zentrale Gehalt des Perfektionismus in dem Gedanken zu liegen, dass Menschen über verbesserbare und steigerbare Eigenschaften und Fähigkeiten ver-

4 Christman (2009), 173; ferner: ,[...] perfectionism, the view that there are ideals of life and human flourishing which can be formulated and defended philosophically and which apply to individuals generally, whether or not they have chosen or accepted such values." (Christman 2009, 14)

5 Siehe die ausführliche Diskussion dieses Problemzusammenhangs bei: Sher (1997), 45-105. 
fügen und dass die Steigerung und Verbesserung dieser Eigenschaften und Fähigkeiten gut und wünschenswert für die betreffende Person ist. Worin diese Vermögen bestehen, in welcher Weise sie entwickelt werden sollten und warum es gut ist, sie zu verbessern, darüber werden die divergierendsten Anschauungen vertreten. Um ein Beispiel für unterschiedliche Antworten auf die Frage nach dem Warum der Selbstverbesserung zu geben: Kant postuliert in der Metaphysik der Sitten und ansatzweise auch schon in der Grundlegung, dass es eine sich aus dem Sittengesetz ergebende moralische Pflicht gebe, die eigenen Talente zu entwickeln ${ }^{6}$; John Stuart Mill meint dagegen, das Interesse an der Kultivierung der höheren Vermögen verdanke sich der Tatsache, dass ihre Ausübung mit qualitativ wertvolleren positiven Erlebnissen (pleasure) verbunden sei (Mill 1861/1969, 210-214). Bei allen Unterschieden waren beide, Kant und Mill, der Auffassung, dass die Sorge um das eigene, hedonistisch verstandene Glück den Selbstverbesserungsanstrengungen Grenzen setzt. In der Grundlegung argumentierte Kant, das Sittengesetz verbiete, die eigenen Begabungen völlig brachliegen zu lassen. Zwischen der völligen Vernachlässigung und der Ausbildung von Höchstformen muss der Einzelne, nach Kants Analyse, moralisch verantwortlich ein richtiges Maß finden - die Selbstvervollkommnung ist als unvollkommene Pflicht ,nach Art und Ausmaß“ unbestimmt. Aus ganz anderen Gründen gelangt Mills Version des ethischen Perfektionismus zu dem Schluss, dass es in den meisten Fällen nicht wünschenswert ist, dass Menschen bei allen sich bietenden Gelegenheiten an ihrer Selbstverbesserung arbeiten. Als Hedonist postuliert er, dass Selbstverbesserungsanstrengungen ihren Wert verlieren, wenn sie nicht mehr mit Freuden (pleasure) verbunden sind oder zu Freuden führen. Wenn der individuelle Sättigungspunkt erreicht ist, sollte man die Tätigkeit wechseln. Ich erwähne dies, um zu veranschaulichen, dass die Kultivierung der Vermögen vor dem Hintergrund völlig unterschiedlicher philosophischer Ansätze gerechtfertigt worden ist, die nichts mit objektiven Wertlehren oder politischem Paternalismus zu tun haben.

\section{Hegel und die Grenzen der Selbstverwirklichung}

Für die in diesem Aufsatz im Vordergrund stehende Frage nach dem Verhältnis von Selbstverwirklichung und Moral konzentriere ich mich auf perfektionistische Entwürfe in der Tradition des Deutschen und Britischen Idealismus. Beginnen möchte ich mit einer Betrachtung Hegelscher Theoriestücke, und zwar aus zwei Gründen: (a) Zum einen entwickelt Hegel die

6 „Es ist ihm [dem Menschen - M. S.] Pflicht: sich aus der Rohigkeit seiner Natur, aus der Tierheit (quod actum), immer mehr zur Menschheit, durch die er allein fähig ist, sich Zwecke zu setzen, empor zu arbeiten: seine Unwissenheit durch Belehrung zu ergänzen und seine Irrtümer zu verbessern, und dieses ist ihm nicht bloß [durch - M. S.] die technisch-praktische Vernunft zu seinen anderweitigen Absichten (der Kunst) anrätig, sondern die moralisch-praktische gebietet es ihm schlechthin und macht diesen Zweck ihm zur Pflicht, um der Menschheit, die in ihm wohnt, würdig zu sein.“ (Kant 1798/1977, 516-517) In der Grundlegung prüft Kant mit der Naturgesetz- (und später mit der Zweck-an-sich-Formel) die Maxime von jemandem, der über ein Talent verfügt, ,welches vermittelst einiger Kultur ihn zu einem in allerlei Absicht brauchbaren Menschen machen könnte“, der es aber vorzieht, lieber „dem Vergnügen nachzuhängen, als sich [um - M. S.] Erweiterung und Verbesserung seiner glücklichen Naturanlagen zu bemühen“" (Kant 1786/1977, 53). Er kommt zu dem Ergebnis, dass die Menschheit zwar bestehen könne, wenn alle ihre Talente brach liegen ließen - allein, sie könnten ihm zufolge unmöglich wollen, dass „dieses ein allgemeines Naturgesetz werde, oder als ein solches in uns durch Naturinstinkt gelegt sei. Denn als ein vernünftiges Wesen will er notwendig, daß alle Vermögen in ihm entwickelt werden, weil sie ihm doch zu allerlei möglichen Absichten dienlich und gegeben sind." (Kant 1786/1977, 53-54) Was Kant hier als unvollkommene Pflicht begründet, könnte man ein Verwahrlosungsverbot nennen. 
bis heute bekannteste Analyse der Selbstverwirklichung und ihrer Pathologien. Es ist wohl kaum übertrieben, zu sagen, dass das Problem der Selbstverwirklichung in der Hegelschen Theorie der Moderne eine zentrale Stellung einnimmt. In dem wichtigen Resümee zum $\S 124$ der Grundlinien der Philosophie des Rechts bezeichnet Hegel das „Recht der Besonderheit des Subjekts, sich befriedigt zu finden“, als den „Wende- und Mittelpunkt in dem Unterschiede des Altertums und der modernen Zeit". Mit diesem Recht der Besonderheit des Subjekts ist das moralische Recht der Individuen gemeint, ihr Leben den eigenen Neigungen und Überzeugungen entsprechend zu gestalten. Hegel erklärt jedoch den moralischen Anspruch der Individuen auf Selbstverwirklichung nicht nur für einen wesentlichen Aspekt des in der Moderne sich realisierenden Geschichtszwecks; er analysiert zudem ausführlich, in welcher Weise dieser Anspruch zu Fehlbildungen führt, wenn er sich nicht philosophisch über sich aufklärt. Diese Fehlbildungen beruhen ihm zufolge im Kern auf einer Verkennung der subjektiven als negativer Freiheit, eine Verkennung, die dialektisch aufgehoben werden muss.

(b) Bei Hegel finden sich aber auch Überlegungen angelegt, die Zweifel an dieser dialektischen Aufhebung aufkommen lassen; in der linkshegelianischen Traditionslinie ist dies in je unterschiedlicher Weise thematisiert worden, etwa beim frühen Marx, wenn er die Abschaffung der Warenförmigkeit der Arbeitskraft und der kapitalistischen Arbeitsteilung als Vorbedingung wahrhafter Selbstverwirklichung anspricht. In der kapitalistischen Gesellschaft sind - dieser Sichtweise folgend - alle Versuche, sich selbst zu verwirklichen, im Ergebnis von den Kräften der Verdinglichung, Ausbeutung und Entfremdung verformt und insofern pathologisch. Wie auch immer man die Überzeugungskraft der jeweiligen Überlegungen einschätzt: Ein wesentliches Verdienst der Hegelschen Tradition besteht darin, die Frage der Selbstverwirklichung vor dem Hintergrund einer entfalteten Theorie der Moderne zu stellen.

Oben hatte ich bereits angedeutet, dass nach Hegel die Moderne durch das „Recht der subjektiven Freiheit" gekennzeichnet ist, das alle gesellschaftlichen Sphären beherrscht und sich immer wieder als reine Negation zur Geltung bringt. ${ }^{7}$ (a) Politisch, insofern die Emanzipation von ständischer Ordnung in die Mordkampagnen des Jakobinismus mündet, den Hegel als den Exzess eines rein negativen Freiheitsverständnisses interpretiert: Die Selbstverwirklichung des modernen politischen Subjekts vollzieht sich zunächst als „Zertrümmerung aller bestehenden gesellschaftlichen Ordnung und [...] Hinwegräumung der einer Ordnung verdächtigen Individuen“" (Hegel 1821/1970, § 5). Nach Hegel kommt hier ein Strukturelement des menschlichen Willens zum Ausdruck - seine Fähigkeit zur Negation, zur Verneinung jeglicher Bestimmung, was ihm erlaubt, den politischen Fanatismus der Jakobiner und den religiösen Fanatismus der indischen Brahmanen auf dieselbe Wurzel zurückzuführen, die „reine Reflexion des Ich in sich“. (b) Sozial führt die moderne Arbeitsteilung und die „Abhängigkeit [des Individuums - M. S.] von äußeren Einwirkungen, Gesetzen, Staatseinrichtungen, bürgerlichen Verhältnissen“ (Hegel 1842/1985, Bd. 1, 151) dazu, dass die Einzelnen das Ganze nicht mehr verkörpern können und müssen, wie dies nach Hegels Auslegung im Athen des Perikles der Fall war. Ihre Rolle im gesellschaftlichen Ganzen ist beschränkt, ihre Bedeutung gemindert. Die modernen Subjekte sind keine „Totalität in sich“, sondern Stückwerk, und sie reagieren auf diese Entwertungserfahrung ihrerseits durch den Rückzug auf das egoistische Einzelinteresse. Hegel diagnostiziert die Egozentrik des modernen Individuums nicht als individuelles Ver-

7 Ausdruckformen dieses modernen Rechts subjektiver Freiheit finden sich ihm zufolge in den Geschlechterbeziehungen (romantische Liebe), im religiösen Leben (protestantische Unmittelbarkeit zu Gott), im Moralverständnis (das eigene Gewissen als alleiniger Maßstab), in den verfassungsmäBig garantierten bürgerlichen Freiheiten, in der Marktsphäre (das „System der Bedürfnisse“) und in der modernen Kunst (siehe Hegel 1821/1970, § 124). 
sagen, sondern als gesellschaftliches Schicksal, das aus der Zumutung einer arbeitsteiligen Produktionssphäre resultiert, die niemandem mehr erlaubt, sich gesellschaftlich arbeitend und handelnd gleichsam selbst zu produzieren (Rechtsphilosophie, § 10, Zusatz). Das Misslingen der Selbstverwirklichung ist somit für Hegel nicht in erster Linie und sicherlich nicht ausschließlich Folge einer falschen Geisteshaltung, sondern nachvollziehbares Resultat eines historischen Entwicklungsstands, der die Verwirklichung des Selbst in bestimmten Hinsichten gar nicht mehr erlaubt. Die Pathologien der Selbstverwirklichung sind immer auch und vor allem Pathologien des Sozialen. Auf diesen Punkt werde ich später nochmals zurückkommen. (c) Ethisch-ästhetisch, insofern Hegel die griechische Skulptur als den höchsten künstlerischen Ausdruck einer gesellschaftlichen und politischen Welt betrachtet hatte, in der die Individuen im sittlichen Ganzen aufgehen. Die Darstellung des schönen Körpers galt ihm als die einer im vernünftigen sittlichen Ganzen aufgehobenen Individualität. Die Schönheit des klassischen Ideals ist jedoch in der Moderne verloren, und „der Geist weiß, daß seine Wahrheit nicht darin besteht, sich in die Leiblichkeit zu versenken“" (Hegel 1842/1985, Bd. 1, 499). Den modernen Individuen, die sich in ihrer Besonderheit zur Geltung bringen wollen, aber sich zugleich als Stückwerk und bedeutungsloses Teil im Ganzen erfahren, erscheint die idealisierte Darstellung schöner Individualität als unwahr, als Kitsch. Die Konflikte antiker Dramen, deren Protagonisten an den inneren Widersprüchen der politischen Welt zu Grunde gehen, die sie mit vollem Pathos verkörpern, werden ihnen zusehends fremd. ${ }^{8}$ Mehr noch: Schönheit wird der Kunst der Moderne, die allenfalls noch für den Betrachter schön gemacht wird, selbst zu einem Äußerlichen und Untergeordneten. Hegel antizipiert hier, was später einmal „abstrakte Kunst" heißen wird. Die geistigen Gehalte der Moderne sprengen das traditionelle Material der schönen Künste, sie zwingen sie, abstrakt und konzeptionell zu werden. Kunst wird ausgestellt, damit die Betrachter sich selbst etwas dazu denken, was die Werke von sich aus weder verbindlich vorgeben können noch wollen. In Figuren wie den Schlegel-Brüdern findet nun nach Hegel die zur völligen Beliebigkeit geratene narzisstische Selbstproduktion des modernen Individuums ihren traurigen Ausdruck. ${ }^{9}$ Die romantische Ironie deutet er als einen in seiner Hohlheit verstörenden Versuch des Individuums, über die Wirklichkeit durch Entwertung aller Gehalte Herr zu werden. Fichtes Lehre vom absoluten Ich autorisiert die romantische Behauptung, dass das „Geltenlassen und Aufheben“ jeglichen Gehalts „rein im Belieben“ des Einzelnen stehe. Carl Schmitt antizipierend, wirft Hegel den Romantikern vor, die Wirklichkeit als bloße Projektionsfläche romantischer Einfälle zu behandeln und den Gedanken der Selbstverwirklichung in ein pathologisches Extrem zu treiben. ${ }^{10}$

8 „Bei den Griechen, bei denen das Pathos, der substantielle Inhalt des Handelns und nicht der subjektive Charakter das Wichtige ist, betrifft das Schicksal weniger diesen bestimmten Charakter, der sich innerhalb seiner Handlung auch nicht wesentlich weiterentwickelt, sondern am Ende ist, was er anfangs war. Auf unserer Stufe aber ist die Fortführung der Handlung [...] eine Weiterentwicklung des Individuums in seinem subjektiven Inneren und nicht nur ein äußerer Fortgang. Das Handeln Macbeths z. B. erscheint zugleich als eine Verwilderung seines Gemüts, mit einer Konsequenz, welche, als die Unentschiedenheit abgeworfen, der Wurf getan ist, durch nichts mehr sich aufhalten läßt." (Hegel 1842/1985, Bd. 1, 554-555)

9 „In der Nachbarschaft nun der Wiedererweckung der philosophischen Idee eigneten sich [...] $\mathrm{Au-}$ gust Wilhelm und Friedrich von Schlegel, nach Neuem in der Sucht nach Auszeichnung und Auffallendem begierig, von der philosophischen Idee soviel an, als ihre sonst eben nicht philosophischen, sondern wesentlich kritischen Naturen aufzunehmen fähig waren." (Hegel 1842/1985, Bd. 1, 71)

10 „Auf dem Standpunkte, auf welchem das alles aus sich setzende und auflösende Ich der Künstler ist, dem kein Inhalt das Bewußtsein als absolut und an und für sich, sondern als selbstgemachter zernichtbarer Schein erscheint, kann solcher Ernst keine Stätte finden, da nur dem Formalismus des Ich 
(d) Moralisch. Hegel versteht die Moral und das moralische Gewissen als ein Phänomen der Moderne, und weicht hier von einem heute verbreiteten Begriffsgebrauch ab. Im Gegensatz zur Sitte, in der die als verbindlich und richtig geltenden Regeln in Gestalt von zugleich unbefragten und sanktionsbewehrten Konventionen und Rechtsnormen auftreten, entspricht die Moral der Forderung moderner Individuen, dass jegliche Einschränkung der subjektiven Freiheit in zugleich zwingender und nachvollziehbarer Weise begründet werde. „Das Recht des subjektiven Willens [...], daß das, was er als gültig anerkennen soll, von ihm als gut eingesehen werde [...]“ (Hegel 1821/1970, § 132; vgl. auch ders. 1830/1970, § 503), macht den Kern dessen aus, was Hegel unter den Begriff Moral fasst. Die Theorie Kants gilt ihm als der avancierteste, aber auf beunruhigende Art zum Scheitern verurteilte Versuch, Pflichten auf eine abstrakte Vorstellung vernünftiger Selbstbestimmung zu gründen und Sitte durch Moral vollständig zu ersetzen. Auf der einen Seite lehrt die Autonomieethik, nur als geltend anzuerkennen, was sie aus sich selbst durch vernünftige Reflexion hervorgebracht hat; auf der anderen Seite erweist sie sich aber als außerstande, aus ihrem formalen Prinzip irgendeinen Inhalt zu gewinnen. „Im Gegenteil kann alle unrechtliche und unmoralische Handlungsweise auf diese Weise gerechtfertigt werden. [...] Daß kein Eigentum stattfindet, enthält für sich ebensowenig einen Widerspruch, als daß dieses oder jenes einzelne Volk, Familie usf. nicht existiere oder daß überhaupt keine Menschen leben." (Hegel 1821/1970, § 135) Hegel ist insofern überzeugt, dass die Moral - in ihrer höchsten Ausformung bei Kant - anomisch wirkt und in letzter Konsequenz zur Aufhebung jeder gerechtfertigten institutionellen Ordnung führen müsste.

Bürgerlicher Egoismus, romantisch-ästhetizistische Innerlichkeit, kantischer Moralismus und politischer Radikalismus sind vier Ausformungen scheiternder Selbstverwirklichung, die Hegel als Symptome einer über sich selbst noch nicht vollends aufgeklärten (und insofern noch nicht zu sich selbst gekommenen) Moderne analysiert. Sie gelten ihm als modernitätsspezifische Entzweiungsphänomene, die aber innerhalb der vom Prinzip der subjektiven Freiheit bestimmten Moderne selbst aufgelöst werden müssen und können. Der Schlüssel zur wahrhaften Selbstverwirklichung liegt ihm zufolge in einem angemessenen Verständnis subjektiver Freiheit. Wissen über die vernünftigerweise zu respektierenden Regeln und Werte erlangt das Individuum nicht durch die inhaltsleere oder willkürliche moralische Reflexion, sondern durch Orientierung an vernünftigen, wirklich geltenden formellen und informellen Institutionen, die Hegel unter die Oberbegriffe „Familie“, „Bürgerliche Gesellschaft“ und „Staat" gruppiert. ${ }^{11}$ Die Individuen verwirklichen sich ihm zufolge wahrhaft selbst, indem sie ihre Aufgabe innerhalb dieser Institutionen wahrnehmen, oder, wie es in den Grundlinien heißt: in ,der Pflicht befreit das Individuum sich zur substantiellen Freiheit“ (Hegel 1821/1970, § 149); sie ist „das Gelangen zum Wesen, das Gewinnen der affirmativen Freiheit“" (Hegel 1821/1970, § 149, Zusatz).

Gültigkeit zugeschrieben ist. - Für andere zwar kann meine Erscheinung, in welcher ich mich ihnen gebe, ein Ernst sein, indem sie mich so nehmen, als sei es mir in der Tat um die Sache zu tun - aber sie sind damit nur getäuscht, pauvre bornierte Subjekte, ohne Organ und Fähigkeit, die Höhe meines Standpunktes zu erfassen und zu erreichen. Dadurch zeigt es sich mir, dass nicht jeder so frei (d. i. formell frei) ist, in allem, was dem Menschen sonst noch Wert, Würde und Heiligkeit hat, nur ein Produkt seiner eigenen Macht des Beliebens zu sehen, in welcher er dergleichen gelten, sich dadurch bestimmen und erfüllen lassen kann oder auch nicht." (Hegel 1842/1985, Bd. 1, 73)

11 „Die sittliche Substanz ist a. als unmittelbarer oder natürlicher Geist, - die Familie; b. die relative Totalität der relativen Beziehungen der Individuen als selbständiger Personen aufeinander in einer formellen Allgemeinheit, - die bürgerliche Gesellschaft; c. die selbstbewußte Substanz als der zu einer organischen Wirklichkeit entwickelte Geist, - die Staatsverfassung." (Hegel 1830/1970, § 517) 
Hegel entwickelt hier den Prototyp dessen, was ich in Abschnitt I das Abhängigkeitsmodell genannt hatte. ${ }^{12}$ Selbstverwirklichung ist für ihn nur denkbar durch den Vollzug eines Systems zu respektierender sittlicher Pflichten. Legt man Christmans Konzept zu Grunde, so ist Hegels Lehre von der Selbstverwirklichung durch ein Leben in substanzieller Freiheit perfektionistisch, da Lebensformen unabhängig von den Urteilen und Neigungen der Einzelnen als gut für diese ausgezeichnet werden. Die Schwäche der Hegelschen Konzeption von Selbstverwirklichung offenbart sich jedoch gerade bei der Frage, weshalb das Individuum ,in der Pflicht [...] seine Befreiung“ (Hegel 1821/1970, § 149) haben soll - also in dem Punkt, der durch das perfektionistische Theorieelement zu beantworten wäre. Die aristotelische Tradition hat diesen Bezug teleologisch, durch eine Lehre von der „Bestimmung des Menschen“ hergestellt; Mill hat dagegen auf hedonistischer Grundlage dafür argumentiert, dass die Entwicklung der höheren Vermögen als Form von Selbstverwirklichung begriffen werden kann, weil sie mit besseren Freuden einhergingen. Dagegen bleibt bei Hegel eine unaufgelöste Spannung bestehen zwischen dem „Recht der Besonderheit des Subjekts, sich befriedigt zu befindet“ (Hegel 1821/1970, § 124), und, beispielsweise, der Rede von der Standesehre als der im „System der Bedürfnisse" waltenden sittlichen Gesinnung - eine Spannung, die einer umstandslosen Gleichsetzung von sittlichem Leben und Selbstverwirklichung entgegensteht. ${ }^{13}$ Dies raubt Hegels Abhängigkeitsmodell einiges an Plausibilität.

Liest man Hegel durch eine linkshegelianische Linse, so finden sich bei ihm indes Spuren einer gegenläufigen Theorie. Dieser, inoffiziellen, Theorie zufolge sollen und können den modernen Individuen die Entzweiungserfahrungen nicht durch Änderungen der gesellschaftlichen Verhältnisse erspart werden - vielmehr gilt es zu begreifen, dass diese Erfahrungen unvermeidlich sind. Mehr als andernorts war Hegel in seinen Vorlesungen über die Ästhetik willens, diese Versöhnung im philosophischen Gedanken, die Einsicht in die Vernünftigkeit moderner Institutionen, als eine subjektiv unbefriedigende Form der Selbstverwirklichung anzusprechen.

Im modernen Staat hat sich das Recht vom Willen des Einzelnen gelöst und institutionell objektiviert, sodass mit der Einsicht in seine Vernünftigkeit immer auch das Bewusstsein verbunden ist, dass es auf einen selbst nicht ankommt. Die erlebte und begriffene objektive Bedeutungslosigkeit des Individuums für die Wirklichkeit des Rechts verhindert, dass die philosophische Versöhnung mit den Institutionen der Moderne, die Einsicht in ihre Vernünftigkeit, als volle Form der Selbstverwirklichung erfahren werden kann ${ }^{14}$; oder, wie es sich in den Vor-

12 Eine Komplikation für diese Behauptung stellt der Umstand dar, dass Hegel von sittlichen und nicht von moralischen Pflichten spricht. Die Lösung liegt darin, dass, was Hegel Sittlichkeit nennt, unter den Begriff der Moral im Sinne des Abhängigkeitsmodells fällt.

13 „Die sittliche Gesinnung in diesem Systeme ist daher die Rechtschaffenheit und die Standesehre, sich, und zwar aus eigener Bestimmung, durch seine Tätigkeit, Fleiß und Geschicklichkeit zum Gliede eines der Momente der bürgerlichen Gesellschaft $\mathrm{zu}$ machen und als solches zu erhalten und nur durch diese Vermittlung mit dem Allgemeinen für sich zu sorgen sowie dadurch in seiner Vorstellung und der Vorstellung anderer anerkannt zu sein.“(Hegel 1821/1970, § 207)

14 Das „Anschließen an die objektive Vernünftigkeit des von der subjektiven Willkür unabhängigen Staates kann entweder eine bloße Unterwerfung sein, weil die Rechte, Gesetze und Institutionen als das Mächtige und Gültige die Gewalt des Zwanges haben, oder es kann aus der freien Anerkennung und Einsicht in die Vernünftigkeit des Vorhandenen hervorgehen, so daß das Subjekt in dem Objektiven sich selber wiederfindet. Auch dann aber sind und bleiben die einzelnen Individuen immer nur das Beiläufige und haben außerhalb der Wirklichkeit des Staates in sich selbst keine Substantialität. [...] Was daher die Einzelnen auch an rechtlichen, sittlichen, gesetzmäßigen Handlungen in dem Interesse und Verlauf des Ganzen vollbringen mögen, ihr Wollen und Ausführen bleibt dennoch wie sie selber immer nur, gegen das Ganze gehalten, unbedeutend und ein bloßes Beispiel.“ (Hegel 1842/1985, Bd. 1, 183) 
lesungen über die Ästhetik in trostloser Härte formuliert findet: „[...] das Allgemeine als solches herrscht in seiner Allgemeinheit, in welcher die Lebendigkeit des Individuellen als aufgehoben oder als nebensächlich und gleichgültig erscheint. “15 Der Trost der Philosophie, die den Individuen die Vernünftigkeit der modernen Institutionen vorführt, bleibt demnach schwach. Die Linkshegelianer, allen voran Marx, forderten denn auch Selbstverwirklichung in einem umfassenden, die Veränderung der gesellschaftlichen Verhältnisse voraussetzenden Sinne ein. Hegels inoffizielle Theorie, der zufolge Philosophie nicht mehr tun kann und soll, als die Individuen zu der Einsicht in die Vernünftigkeit der bestehenden Verhältnisse zu führen und sie so mit ihnen zu versöhnen, wäre demnach keine Version des Abhängigkeitsmodells. Vielmehr wäre sie die schroffe These, dass der moderne Anspruch auf Selbstverwirklichung zugleich anerkannt und in seiner Unerfüllbarkeit verstanden und so aufgehoben werden muss.

\section{Theunissens Unabhängigkeitsmodell}

Nach Hegels offizieller Theorie verwirklicht sich das moderne Individuum wahrhaft selbst in der angemessenen Teilhabe an den vernünftigen Institutionen und Interaktionszusammenhängen von Familie, Wirtschaft und Staat. Die unterschiedlichen Pathologien der Selbstverwirklichung - politischer Radikalismus, kantischer Moralismus, romantisch-ästhetizistische Innerlichkeit, bürgerlicher Egoismus - führt er auf ein unzureichendes Verständnis des Prinzips der subjektiven Freiheit zurück. Indem jedoch das Individuum die Institutionen- und Interaktionsordnung als vernünftig begreift, ist es in die Lage versetzt, seine Teilhabe als Form der Selbstverwirklichung und damit als ,positive Freiheit“" zu verstehen und zu erleben.

Die These der offiziellen Theorie, dass Selbstverwirklichung durch den Verlust subjektiv realisierter Allgemeinheit in die Krise gerät, ist jedoch nicht zuletzt in der existenzphilosophischen Tradition vehement bestritten worden, der zufolge Selbstverwirklichung eine „gewisse Distanzierung von anderen“ und anderem einschlösse und keineswegs einen Allgemeinheitsbezug verlange. ${ }^{16}$

Träfe dies zu, so wäre interessanterweise auch Hegels inoffizielle Theorie zur Disposition gestellt, Selbstverwirklichung sei im modernen Staat nur in dem eingeschränkten Sinne möglich, dass die Individuen die Vernünftigkeit des Bestehenden begriffen und sich so mit ihren Erfahrungen von Entfremdung und Entzweiung versöhnten. Wenn Selbstverwirklichung, ganz im Gegenteil, die Distanzierung von der Zumutung voraussetzt, das Allgemeine subjektiv zu realisieren, so gewinnt die These an Plausibilität, erst im modernen Staat sei es den Individuen möglich geworden, die Verwirklichung ihrer selbst überhaupt zu denken. Denn er ermöglicht ihnen genau jene Entgegensetzung zum oder Abkehr vom Allgemeinen, die aus existenzphilosophischer Sicht Voraussetzung des ,Selbstseins“ ist.

Vor diesem Hintergrund argumentiert Michael Theunissen in seiner „Kritik des gegenwärtigen Bewusstseins“, dass der Allgemeinheitsbezug keine Voraussetzung gelingender Selbstverwirklichung sei, sondern eine Forderung der Moral darstelle. Er macht sich zu Eigen, was in Abschnitt I das Unabhängigkeitsmodell genannt wurde.

Theunissen ist überzeugt, dass $\mathrm{zu}$ den geistesgeschichtlichen Voraussetzungen von Abhängigkeitsmodellen eine verbindliche Vorstellung von der „Bestimmung des Menschen“

15 Hegel (1842/1985), Bd. 1, 184 (Hervorhebung von mir - M. S.). Mir scheint dieses „oder“ erläuternd zu sein.

16 Theunissen (1982), 13. 
gehört habe und dass diese Voraussetzung spätestens seit Mitte des 19. Jahrhunderts unwiederbringlich verloren gegangen sei. Dass die Moderne nicht mehr wisse, was der Mensch sei und wozu er bestimmt wäre, und dass die Aufgabe von festgelegten Meinungen über die Bestimmung des Menschen das gültige Ergebnis eines historischen Lernprozesses darstelle, ist eine Einschätzung, die Theunissen mit der Existenzphilosophie teilt. Der „Modernismus“ distanziert sich von der Möglichkeit, „Selbstverwirklichung als Menschwerdung“ aufzufassen, und er tut dies zu Recht, wenn auch mit zuweilen moralisch kritikwürdigen Konsequenzen. ${ }^{17}$ Der moderne, auf die je eigene Individualität gerichtete Anspruch auf Selbstverwirklichung erscheint bei Theunissen als das Endresultat des Zerfallsprozesses von Religion und Metaphysik. Es kommt zu einer alle Möglichkeiten offen lassenden „Reduktion der Selbstverwirklichung auf eine Verwirklichung der je eigenen Individualität““. ${ }^{18}$ Das für die philosophische Tradition seit Platon und Aristoteles Undenkbare erscheint nun möglich: dass ein moralisch verdorbener Mensch in einem vollen Sinne sich selbst verwirklicht. Für Theunissen drängt sich damit die Frage auf, wie Selbstverwirklichung unter Bedingungen der modernen Bestimmungslosigkeit des Menschen „moralisch eingeholt“ werden kann. Die Suche nach Antworten bei Hegel aufzunehmen, liegt für ihn unter anderem deshalb nahe, weil Hegel einerseits postuliere, der Einzelne habe „ein allgemeines Leben zu leben“, andererseits aber davon ausgehe, dieses Allgemeine sei nicht durch eine Lehre über die aus der Bestimmung des Menschen folgenden Tugenden vorgegeben.

Freilich ist, was Theunissen als Begründung der ersten der beiden Thesen von Hegel übernimmt, äußerst dürftig ausgefallen: „Wenn ich Ich sage, so meine ich mich als diese einzelne, durchaus bestimmte Person. In der Tat sage ich jedoch dadurch nichts Besonderes von mir aus. Ich ist auch jeder andere, und indem ich mich als Ich bezeichne, so meine ich zwar mich, diesen Einzelnen, spreche jedoch zugleich ein vollkommen Allgemeines aus." (Hegel 1830/1970, § 24, Zusatz 1) Dass sehr viele Menschen sich auf sich selbst mit dem Wort „Ich“ beziehen, bedeutet freilich nicht, dass das Wort etwas vollkommen Allgemeines ausspricht. Wenn man ruft „Ich bin es leid!“”, dann meint man sich ,,als diese einzelne, durchaus bestimmte Person“" und sagt - entgegen Hegels Behauptung - durchaus etwas Besonderes über sich aus, nämlich, dass man es leid ist. Es kann zwar sein, dass jemand, der einen dies rufen hört, nicht erkennen kann, wer da ruft, aber dies ändert nichts daran, dass der Satz Informationsgehalt hinsichtlich einer ganz bestimmten Person hat, auch wenn andere vielleicht nicht bestimmen können, wer diese Person sei. Aus dem Umstand, dass sehr viele Menschen „Ich bin es leid!“ rufen können oder gerufen haben könnten, folgt nicht, dass der Ausruf „ein vollkommen Allgemeines“ ausspricht, wie Hegel behauptet. „Ich bin es leid!“ bestimmt die rufende Person und niemanden sonst. ${ }^{19}$ Damit entfällt aber die von Theunissen im Anschluss an Hegel angestrebte Pointe, dass es eine „Dialektik des Ich-Sagens“ gebe, der zufolge Selbstbestimmung in einem tiefen metaphysischen Sinne in Allgemeinheit übergehe

17 Ebd., 6.

18 Ebd., 7.

19 „Die deiktischen Ausdrücke [,,hier“, ,,jetzt“, ,,dieses“, ,ich“- M. S.] unterscheiden sich [...] von den anderen singulären Termini [das heißt Termini, die einen einzelnen Gegenstand bezeichnen - M. S.] dadurch, dass von der Sprechsituation abhängt, welcher Gegenstand jeweils mit ihnen bezeichnet wird." (Tugendhat 1979, 71) Es ist nur konsequent, dass Hegel einen anderen deiktischen Ausdruck („diese(s)“) in der Phänomenologie des Geistes in analoger Weise analysiert. Die „Dialektik der sinnlichen Gewißheit“ besteht nach Hegel darin, dass das „Dieses nämlich als ein Allgemeines“ (Hegel 1806/1970, 90-91) offenbar wird. Beiden Analysen scheint aber ein unzureichendes Verständnis des Charakters deiktischer Ausdrücke zu Grunde zu liegen. 
oder an Allgemeinheit gebunden sei. ${ }^{20}$ Und es ist unklar, was Theunissen damit meint, dass es zum „Verständnis meiner selbst als eines Ichs“ wesentlich hinzugehöre, dass man im IchSagen zu sich sage, „was alle Subjekte als solche zu sich sagen“. ${ }^{21}$

Dieser Versuch, den Allgemeinheitsbezug einer „ethisch qualifizierten Selbstverwirklichung“ unter Verzicht auf die „Bestimmung des Menschen“ philosophisch zu begründen, hängt in der Luft.

erscheint ein anderer, ebenfalls bei Hegel angelegter Ansatz, dem zufolge Individuen über das Eintreten in Interaktions-, Bedeutungs-, Norm- und Wertsysteme ,,sozial konstituiert“" werden. Wenn das Individuum sozial konstituiert ist, drängt es sich auf, Selbstverwirklichung als etwas zu begreifen, was sich innerhalb und durch soziale Beziehungen vollzieht. Freilich fragt sich, wie weit dieser Ansatz für das Projekt einer Moralisierung der Selbstverwirklichung trägt. Die für Intersubjektivität vorausgesetzten Normen und Werte sind nicht unbedingt moralisch angemessen. Entsprechend stellt die soziale Natur des Menschen nicht sicher, dass die Individuen eine moralisch akzeptable Vorstellung davon haben, wie sie sich selbst in sozialen Beziehungen verwirklichen wollen. Um die moralische Angemessenheit sicherzustellen, zieht Theunissen noch einmal die dialektische Struktur des Ich-Sagens heran, von der er nun behauptet, dass sich aus ihr der Gedanke einer „Allheit gleicher Subjekte“ gewinnen lasse. Dass ihm zufolge die Analyse des Ich-Bewusstseins zum Gleichheitsgedanken führt, nutzt er für die Bestimmung derjenigen sozialen Bezüge, in denen sich das Selbst in moralisch akzeptabler Weise verwirklichen kann. Zur moralischen Verwirklichung ihrer selbst gelangt eine Person, wenn sie in ihren Beziehungen allen Anderen als Gleichen gerecht wird. Der Gleichheitsgedanke wiederum gibt die vernünftigen Projekte vor, die eine in moralischer Weise sich verwirklichende Person verfolgen wird: Die Selbstverwirklichung, ,die von uns gefordert ist, konkretisiert sich heute [...] in der Bekümmerung um die weltweite Ausbeutung der Natur, in der Betroffenheit vom Hunger in der Welt, in der Sorge um den Weltfrieden". ${ }^{22}$

Wie sind nun Theunissens Überlegungen mit Blick auf die oben eingeführte Unterscheidung zwischen einem Vereinbarkeits- und einem Unvereinbarkeitsmodell der Selbstverwirklichung einzustufen? In Übereinstimmung mit der Existenzphilosophie geht Theunissen davon aus, dass es nicht-moralische Formen, sich selbst zu verwirklichen, geben kann. Dafür, dass es ihm um ein Vereinbarkeitsmodell zu tun ist, spricht nun zum einen, dass er den Allgemeinheitsbezug im Sinne der „Allheit gleicher Subjekte“ im Ich-Bewusstsein angelegt sieht. Zum anderen schreibt er, das Glückskriterium sei ,allein dann rechtmäßig, wenn es so gefasst wird, dass es das abstrakt individuelle Glück ausschließt, das als seinen Preis das Unglück der anderen verlangt“. ${ }^{23}$ In diesem Zusammenhang unterscheidet er das „In-Frieden-mit-sich-Sein“von „,bloßer Zufriedenheit“, wobei das „In-Frieden-mit-sich-Sein“ eine eigene Form des Glücks darzustellen scheint, die mit der moralischen Selbstverwirklichung einhergeht. Doch Theunissen kann nicht plausibel machen, wie seine Forderung nach einer Remoralisierung des Konzepts, die bei aller Bemühung um eine philosophisch abstrakte Grundlegung doch unverkennbar vom Zeitgeist der 1980er Jahre geprägt ist, auf den berechtigten Anspruch des Individuums antwortet, nicht bloß

20 „Hegel begründet seinen Gedanken seinerseits vornehmlich mit dem Hinweis auf die Dialektik des Ich-Sagens. Sage ich ,ich', so meine ich mich als diesen einzelnen und zugleich die schwer fassbare Sache, die alle meinen, wenn sie ,ich' sagen. Diese Struktur zeichnet formal vor, was in der Bildung subjekthafter Einzelheit oder in ethisch qualifizierter Selbstverwirklichung eigentlich zu verwirklichen ist.“" (Theunissen 1982, 19)

21 Ebd., 22.

22 Ebd., 46.

23 Ebd., 48. 
Instrument der Verwirklichung moralischer Zwecke zu sein. Der Verweis auf die angebliche „Dialektik des Ich-Sagens“ ist sicherlich unzureichend, um die Interpretation moralischen Handelns als selbstverwirklichend abzustützen. Gibt man diesen Punkt auf, so bleibt in Theunissens Modell die These, dass die berechtigten Ansprüche auf Selbstverwirklichung durch die berechtigten Ansprüche anderer begrenzt werden müssten, ohne dass von dieser Begrenzung gesagt werden könnte, in ihr verwirkliche man sich selbst.

\section{Selbstverwirklichung und "wahres Selbst"}

Einen ehrgeizigen und im deutschen Sprachraum wenig bekannten Versuch, Moral als notwendige Voraussetzung der Selbstverwirklichung zu denken, geht auf Thomas Hill Greens Prolegomena to Ethics zurück, einem Hauptwerk des Britischen Idealismus. In vielen Hinsichten steht Greens Entwurf Hegel, Fichte und Kant nahe, doch ist in seinen Überlegungen zur Selbstverwirklichung auch der Einfluss eingehender Aristoteles-Lektüren merklich.

Ich hatte Abschnitt II mit der Bilanz geschlossen, dass Hegels offizielle Theorie die Erklärung dafür schuldig bleibe, warum man das Aufgehobensein in den Institutionen und Interaktionsformen moderner Gesellschaften als Selbstverwirklichung begreifen könne; als Beispiel hatte ich auf die Spannung zwischen dem Recht auf Besonderheit und der sittlichen Idee der Standesehre verwiesen. Wenn das Individuum in den sittlichen Verhältnissen seine Verwirklichung finden soll, bleibt für Eigenheit, Eigensinn, Kreativität und Erfindungsgeist nur wenig Raum. ${ }^{24}$ Was Mill „Lebensformexperimente“ genannt hat, liegt wohl außerhalb dessen, was Hegel an Besonderheit zuzulassen bereit war. ${ }^{25}$

Hegels inoffizielle Theorie läuft dagegen auf die ernüchternde Diagnose hinaus, dass Selbstverwirklichung in einem emphatischen Sinne in der modernen Gesellschaft nicht möglich ist. Die Individuen der Moderne sind keine ,ganzen Menschen“ mehr. ${ }^{26}$ Gleichwohl ist die gesellschaftliche Welt, in der sie sich vorfinden, das vernünftige und zustimmungsfähige Endergebnis einer gerichteten historischen Entwicklung. So lässt sich nach Hegel die Entzweiung nicht wieder zurücknehmen, aber doch in ihrer Notwendigkeit verstehen und in diesem Sinne aufheben. Diese dialektische Bewegung macht die Menschen der Moderne allerdings nicht wieder ganz. Sie bleiben Stückwerk, und Philosophie vermag nicht mehr, als sie mit der Unmöglichkeit voller Selbstverwirklichung zu versöhnen.

Man kann nun Greens Entwurf in den Prolegomena als einen Vorschlag lesen, mit dem sich sowohl das Begründungsdefizit der offiziellen Theorie als auch die deprimierende Diagnose der inoffiziellen Theorie Hegels vermeiden lässt.

24 In einer erhellenden Auslegung hat Ludwig Siep gemeint, nach Hegel „würde Selbstverwirklichung in sozialen Rollen erfordern, diesen Rollen und Funktionen den eigenen Stempel aufzudrücken, sie - analog zur Inszenierung von Kunstwerken - nach der eigenen Einsicht in das Notwendige und Zeitgemäße, auf persönliche, in Maßen auch innovatorische Weise zu ,inszenieren“" (Siep 2002, 50). Selbstverwirklichung scheint aber mehr als dies zu erfordern.

$25 \mathrm{Zu}$ den Millschen Lebensformexperimenten: Kuenzle u. Schefczyk (2009), $177 \mathrm{ff}$.

„Bei Achill kann man sagen: das ist ein Mensch; die Vielseitigkeit der edlen menschlichen Natur entwickelt ihren ganzen Reichtum an diesem einen Individuum. Und so ist es auch mit den übrigen Homerischen Charakteren: Odysseus, Diomed, Ajax, Agamemnon, Hektor, Andromache; jeder ist ein Ganzes, eine Welt für sich, jeder ein voller, lebendiger Mensch und nicht etwa nur die allegorische Abstraktion irgendeines vereinzelten Charakterzuges.“ (Hegel 1842/1985, Bd. 1, 234) 
John Skorupski merkt in einem Aufsatz an, dass Green einen wichtigen Platz in der Galerie der idealistischen Philosophen einnimmt, denen es im Kern um die Frage ging, wie Individuen in modernen Gesellschaften ein nicht-entfremdetes und spirituell erfüllendes Leben führen können (Skorupski 2006, 47). Doch Greens philosophische Herangehensweise unterscheidet sich grundlegend von derjenigen Hegels. Er bettet seine Theorie der Selbstverwirklichung nicht - wie Hegel - in eine (ihrerseits in eine Geschichtsmetaphysik eingelagerte) Modernetheorie ein. Seine Überlegungen sprechen daher Phänomene wie Entfremdung und Entzweiung nicht direkt an. Schließt man sich ihnen jedoch an, so verliert Hegels inoffizielle Theorie, dass moderne Individuen keine ,ganzen Menschen“, sondern Stückwerk seien, an Dramatik. Für Green beruht nämlich eine solche Diagnose auf einer metaphysischen Voraussetzung, die er für falsch hält, namentlich, dass Personen in einem dramatischen Sinne ,getrennt“ seien. Um es in Variation eines Bonmots von Adorno zu sagen: Personen sind nach Green getrennt, aber so getrennt auch wieder nicht. Das Wohlergehen anderer ist in einem bedeutenden Sinne Bestandteil des eigenen Wohlergehens. Dies erlaubt Green vieles, was in der Hegelschen Tradition als Entfremdung, Entzweiung und Instrumentalisierung erscheinen mag, als Form der Verwirklichung des wahren Selbst anzusprechen.

Greens Theorie ist komplex, und ich werde mich im Folgenden auf einen Gedankenstrang beschränken. Ansatzpunkt ist die Überlegung, dass menschliches Handeln sich nicht einfach aus dem jeweils stärksten Wunschimpuls ergibt, wie Mill meinte ${ }^{27}$, sondern von der „Autorisierung" durch den Akteur abhängt. Menschen verfügen über das Vermögen, ihr Handeln in Abhängigkeit von ihren Urteilen über die Richtigkeit des Tuns zu steuern. Die Instanz, der dieses Vermögen der deliberativen Steuerung zugeschrieben wird, ist das Selbst. Wunschimpulse benötigen die (implizite oder explizite) Autorisierung durch das Selbst, um im Handeln wirksam zu werden. Es macht sich den Impuls durch die Autorisierung zu Eigen, identifiziert sich mit ihm (Green 1883/2003, § 102). Durch die Autorisierung von und die Identifikation mit Wünschen bestimmt sich das Selbst; im Handeln verwirklicht sich diese Bestimmung. Nun setzt die Beurteilung von Wünschen durch das Selbst dessen Fähigkeit voraus, eine Vorstellung des Guten vorzugeben (Green 1883/2003, § 220). Diese Vorstellung des Guten bezieht sich auf den Gedanken von sich als einem zeitlich überdauernden Wesen. Wünsche werden vom deliberierenden Selbst mit Blick auf die Frage beurteilt, ob sie zu demjenigen beitragen, was ,gut für einen selbst“ ist. Zusammenfassend: Das Selbst ist eine deliberative Instanz, die einen Begriff vom Lebensganzen bildet und die Menge vergänglicher mentaler Zustände zu einer Einheit zusammenfasst; die Bewertung der Wünsche geschieht mit Blick auf deren Stellung innerhalb dieser Einheit. ${ }^{28}$

Der Gedanke von der Einheit der Person spricht nach Green entschieden gegen den Hedonismus. Was gut für eine Person im Ganzen ist, kann sich nicht als die Summe der Befriedigung einzelner Wünsche ergeben. Denn Bedürfnisbefriedigungen lassen sich nicht zu Gesamtsummen aufaddieren (Green 1883/2003, § 227). Wir könnten zwar so reden, als sei die Menge an Lust in $A$ 's Leben insgesamt größer als die Menge an Lust in $B$ 's Leben, aber wir dürften dabei nicht vergessen, dass Lustzustände nicht persistierten. Insofern ist die Maximierung der Gesamtlustmenge im Lebensverlauf kein sinnvoller Kandidat, wenn man

27 Mill (1865/1979), 453

28 Wenn das Selbst die Zulässigkeit von konativen Zuständen beurteilt, dann beschränkt es sich nicht auf Abwägungen zwischen jeweils konfligierenden Neigungen oder Abschätzungen der Zweckrationalität hinsichtlich eines akuten Wunsches. Es beurteilt Wünsche vielmehr hinsichtlich ihrer Rolle für die Einheit der Person: ob sie diese Einheit stören oder fördern. Ich sehe hier Bezüge zu Julian Nida-Rümelins Konzept der strukturellen Rationalität (Nida-Rümelin 2001). 
wissen will, was ,gut für einen selbst“ ist (Green 1883/2003, § 229). Das für einen selbst Gute muss das eigene Leben als Ganzes betreffen und daher selbst Wirklichkeit und Dauer haben.

Selbstverwirklichung bedeutet nun nach Green die Objektivierung derjenigen Einheit und Dauer, die das Selbst als deliberative Instanz auszeichnet. Solche Objektivierung vollzieht sich in der Verfolgung von beständigen, dem Leben Einheit gebenden Gütern (,,permanent goods“). Green vermutet, dass historisch die Familie das erste „,beständige Gut“ war, das den Menschen ermöglicht hat, das eigene Leben als Einheit zu verstehen und zu fördern. ${ }^{29}$ Er formuliert dies tentativ (Green 1883/2003, § 230), betont aber, dass die Familie die Eigenschaften erfüllt, die gegeben sein mussten, damit sich eine Vorstellung von der beständigen Einheit des Selbst überhaupt bilden konnte: Es brauchte einen Gegenstand, mit dem einerseits starke natürliche Wünsche verbunden sind, der andererseits aber die Dauerhaftigkeit besitzen kann, die nötig ist, damit das Selbst sich in einem Objekt zu realisieren vermag. Die Familie als Institution verdankt sich somit nach Green dem Interesse an einem beständigen Gut, ein Interesse, das mit dem erwachenden Bewusstsein des eigenen Selbst als einer zeitlich erstreckten Instanz einhergeht. ${ }^{30}$ Green verallgemeinert diese These: Was wir anstreben, indem wir ein beständiges Gut für uns selbst wollen, ist ein gemeinsames Gutes. ${ }^{31}$ Das Bewusstsein seiner selbst als einer dauerhaften Instanz einerseits und das Bewusstsein eines gemeinsamen Gutes andererseits sind ihm zufolge ,gleichursprünglich“. Selbstverwirklichung vollzieht sich entsprechend durch die Teilhabe an einer Gemeinschaft mit einem gemeinsamen Gut. Eine Person, die in einer ruhigen Stunde darüber nachdenkt, worin ihr Wohlergehen wirklich besteht, braucht nach Green diese Zusammenhänge nicht philosophisch zu durchschauen - sie wird dies in aller Regel auch nicht können. Wenn sie nicht durch schlechte Philosophie, namentlich den Hedonismus, verdorben ist, wird sie vielmehr ihre Selbstverwirklichung unmittelbar in derjenigen Lebensordnung suchen, die sie umgibt - und die durch jene Zusammenhänge letztlich erklärt wird. ${ }^{32}$ Indem sie ihre Selbstverwirklichung in einem Beitrag zum gemeinsamen Guten findet, verliert die Unterscheidung zwischen dem eigenen Wohlergehen und dem der Anderen ihren Halt. ${ }^{33}$ Die Anderen, mit denen wir in wesentlicher (die Einheit des Selbst verwirklichender) Gemeinschaft leben, sind unsere alter-egos, deren Wohlergehen

29 „In those forms of human life which we can know, either from the intercourse of present society or from the record of the past, this object has probably had the largest share in filling up the idea of true or permanent good.“ (Green 1883/2003, § 229)

30 Es ist vermutlich als Spitze gegen bestimmte Auslegungen der darwinistischen Abstammungslehre zu lesen, wenn Green anmerkt: „From beings incapable of such an interest [in permanent good M. S.], even though connected by acts of generation with ourselves, we cannot in any intelligible sense have been developed. They cannot have had any such essential community with ourselves as would be implied in calling them men." (Green 1883/2003, § 231)

31 ,If this answer is accepted to the question, what it is that we desire in desiring our own true or permanent well-being it would seem that we have already answered the question, what it is that we desire in desiring the true well-being of others. It is the same common well-being, the same good of a society which we also desire as our own." (Green 1883/2003, § 235)

32 „The thought of his well-being will be to him the thought of himself as living in the successful pursuit of various interests which the order of society - taking the term in its widest sense - has determined for him; interests ranging, perhaps, from provision for his family to the improvement of the public health or to the production of a system of philosophy. The constituents of the contemplated well-being will be the objects of those various interests, objects $(e . g$. the provision for a family or the sanitation of a town) in process of realization, which, when realised, take their place as permanent contributions to an abiding social good." (Green 1883/2003, § 234)

33 „The opposition of self and others does not enter into the consideration of a well-being so constituted.“ (Green 1883/2003, § 235) 
einen konstitutiven Teil unseres eigenen Wohlergehens ausmacht (Green 1883/2003, §§ 191, 200). Green behauptet sogar, dass zwischen dem Ego und dem Alter-Ego kein wahrhafter Interessenkonflikt bestehen kann.

Indem er den Gehalt der Moral aus einer philosophischen Idee von Selbstverwirklichung gewinnen will, legt sich Green auf eine extreme Version des Abhängigkeitsmodells fest. Personen können moralischen Anforderungen gerecht werden, weil und nur weil sie sich darin selbst verwirklichen. Ihr Interesse an Einheit und Dauer des eigenen Lebens, an Selbstverwirklichung, kann nur in der Verfolgung gemeinsamer Güter erfüllt werden. Aus diesem Grund firmiert Greens Position als metaphysischer Egoismus. Das Gedeihen des Gemeinsamen ist auch eigenes Gedeihen, der eigene Beitrag zum Wohlergehen aller ist auch Beitrag zum eigenen Wohlergehen.

Kommen wir zu der Frage zurück, ob Greens Entwurf sowohl das Begründungsdefizit der offiziellen Theorie als auch die deprimierende Diagnose der inoffiziellen Theorie Hegels vermeiden kann. Um mit Letzterem zu beginnen, verschiebt sich bei Green der Akzent auf interessante Weise. Während Hegels inoffizielle Theorie die Objektivierung des Rechts und die hochgradige gesellschaftliche Arbeitsteilung als Entfremdungsursachen anspricht (eine Sicht, die im Linkshegelianismus ins Zentrum gerückt wurde) $)^{34}$, meint Green, dass beides mit der Verwirklichung des wahren Selbst vereinbar ist. Moderne Individuen brauchen die Einheit ihres Selbst durch das System der Arbeitsteilung nicht bedroht zu sehen, denn in diesem System verwirklichen sich gemeinsame Ziele, die ihre eigenen sind. Projekte, wie die Verbesserung der öffentlichen Gesundheit, die Formulierung eines philosophischen Systems oder die Sorge um Kinder, sind Beiträge zu einem beständigen sozialen Gut, in dem das Selbst zu eigener Beständigkeit findet (Green 1883/2003, § 234). Entfremdung droht in dieser Sicht nicht durch den Ein-, sondern durch den Ausschluss vom System der Arbeitsteilung, in der Verweigerung der Teilhabe durch politisch strukturierte kapitalistische Märkte. Greens Überlegungen bleiben aber zu abstrakt und unbestimmt, um sagen zu können, ob ihm zufolge der freiwillige oder erzwungene Rückzug aus dem System der Erwerbsarbeitsteilung durch andere Formen der sozialen Vereinigung ausgeglichen werden kann. Davon unabhängig scheint aber sein Grundgedanke plausibel, dass Selbstverwirklichung nicht dadurch unmöglich wird, dass man nicht „heute dies, morgen jenes“ tun kann, wie es in der Deutschen Ideologie heißt.

34 „Sowie nämlich die Arbeit verteilt zu werden anfängt, hat Jeder einen bestimmten ausschließlichen Kreis der Tätigkeit, der ihm aufgedrängt wird, aus dem er nicht heraus kann; er ist Jäger, Fischer oder Hirt oder kritischer Kritiker und muß es bleiben, wenn er nicht die Mittel zum Leben verlieren will - während in der kommunistischen Gesellschaft, wo Jeder nicht einen ausschließlichen Kreis der Tätigkeit hat, sondern sich in jedem beliebigen Zweige ausbilden kann, die Gesellschaft die allgemeine Produktion regelt und mir eben dadurch möglich macht, heute dies, morgen jenes zu tun, morgens zu jagen, nachmittags zu fischen, abends Viehzucht zu treiben, nach dem Essen zu kritisieren, wie ich gerade Lust habe, ohne je Jäger, Fischer, Hirt oder Kritiker zu werden. Dieses Sichfestsetzen der sozialen Tätigkeit, diese Konsolidation unsres eignen Produkts zu einer sachlichen Gewalt über uns, die unsrer Kontrolle entwächst, unsre Erwartungen durchkreuzt, unsre Berechnungen zunichte macht, ist eines der Hauptmomente in der bisherigen geschichtlichen Entwicklung, und eben aus diesem Widerspruch des besondern und gemeinschaftlichen Interesses nimmt das gemeinschaftliche Interesse als Staat eine selbständige Gestaltung, getrennt von den wirklichen Einzel- und Gesamtinteressen, an, und zugleich als illusorische Gemeinschaftlichkeit, aber stets auf der realen Basis der in jedem Familien- und Stamm-Konglomerat vorhandenen Bänder, wie Fleisch und Blut, Sprache, Teilung der Arbeit im größeren Maßstabe und sonstigen Interessen - und besonders, wie wir später entwickeln werden, der durch die Teilung der Arbeit bereits bedingten Klassen, die in jedem derartigen Menschenhaufen sich absondern und von denen eine alle andern beherrscht.“ (Marx u. Engels 1846/1978, 33) 
Das „Festsetzen der sozialen Tätigkeit, die Konsolidation unseres eigenen Produkts“ ist für Green, im Gegenteil, Voraussetzung für Selbstverwirklichung.

Nun zu der Frage, ob Green besser als Hegel plausibel machen kann, warum man sagen kann, dass sich das Selbst durch die Übernahme sozialer Rollen selbst verwirklicht: Der Einwand gegen Hegel lautete, dass bei ihm das „Recht auf Besonderheit“ nur innerhalb vorgegebener sozialer Rollen - und insofern verkürzt und verformt - zur Geltung kommt. Hegels Kerngedanke, dass „Selbstverwirklichung als Vorgang des ,Sich-eine-Wirklichkeit-Gebens“ in der Welt" (Jaeggi 2005, 184) zu verstehen ist; und dass diese Welt eine soziale Welt sein muss, bleibt dagegen unbestritten. Mir scheint nun der wesentliche Vorzug von Greens Herangehensweise, dass seine Metaphysik von dem Selbst als einer deliberativen, Einheit stiftenden und Verwirklichung anstrebenden, Instanz ausgeht. Dies erlaubt, offen zu lassen, welche Gestalt das beständige Gute und die entsprechende Gemeinschaft im Leben des Einzelnen hat. Der Verzicht auf eine metaphysisch begründete, schematische Vorstellung vernünftiger sozialer Ordnung, in der Einzelne ihren Platz einzunehmen haben, lässt Green weniger anfällig für die Kritik werden, dass er keinen Raum lasse für das von Hegel proklamierte, aber dann doch wieder kassierte Recht auf Besonderheit. Greens konstruktivistische Vorstellung des Selbst ist abstrakt und allgemein genug, um dem Kerngedanken gerecht zu werden, dass es bei der Selbstverwirklichung darum gehe, sich in der sozialen Welt Wirklichkeit zu geben; weil dieser Gedanke zugleich weitgehend offen lässt, in welchen sozialen Formen dies geschieht, ist das Konzept im Prinzip offen genug, um auch Millsche Lebensexperimente als Formen von Selbstverwirklichung zuzulassen.

Doch überfrachtet Green diese Idee in moralistischer Manier, weil der angemessen gefasste Begriff der Selbstverwirklichung zugleich alle Fragen moralischer Richtigkeit lösen soll. Dies wiederum zwingt ihn dazu, die Möglichkeit von genuinen Konflikten zwischen Individuen mit Blick auf ihre Rechte, Interessen und Konzeptionen des Guten schlichtweg in Abrede zu stellen und die Einheit und Harmonie zwischen ihnen maßlos zu übertreiben. Mit Recht hat John Skorupski angemerkt, dass die Sehnsucht nach Versöhnung, die für die Ethik des 19. Jahrhunderts so prägend war, eine Tür zum Totalitarismus des 20. Jahrhunderts geöffnet hat. ${ }^{36^{*}}$

Prof. Dr. Michael Schefczyk, Leuphana Universität Lüneburg, Institut für Kulturtheorie, Kulturforschung, Künste, Lehrstuhl Praktische Philosophie, Scharnhorststraße 1, 21335 Lüneburg

\section{Literatur}

Brink, D. (2003), Perfectionism and the Common Good. Themes in the Philosophy of T. H. Green, Oxford.

Christman, J. (2009), The Politics of Persons: Individual Autonomy and Socio-historical Selves, New York.

Green, T. H. (1883/2003), Prolegomena to Ethics, New Edition with Introduction by David Brink, Oxford.

Jaeggi, R. (2005), Entfremdung. Zur Aktualität eines sozialphilosophischen Problems, Frankfurt/M.

Hegel, G. W. F. (1806/1970), Phänomenologie des Geistes, in: ders., Theorie Werkausgabe, Bd. 3 , Frankfurt/M.

36 Skorupski (2006), 50-51 (Fn.); vgl. auch Brink (2003), 60 ff.

* Für Kritik und Anregungen danke ich Inge Gmelin und Christoph Henning. 
Ders. (1821/1970), Grundlinien der Philosophie des Rechts, in: ders., Theorie Werkausgabe, Bd. 7, Frankfurt/M.

Ders. (1830/1970), Enzyklopädie der philosophischen Wissenschaften im Grundrisse, in: ders., Theorie Werkausgabe, Bd. 8-10, Frankfurt/M.

Ders. (1840/1970), Vorlesungen über die Philosophie der Geschichte, in: ders., Theorie Werkausgabe, Bd. 12, Frankfurt/M.

Ders. (1842/1985), Vorlesungen über die Ästhetik, 2 Bde., Berlin.

Hurka, T. (1993), Perfectionism, New York.

Kant, I. (1786/1977), Grundlegung zur Metaphysik der Sitten, in: ders., Werkausgabe, Bd. 7, Frankfurt/M., 7-102.

Ders. (1798/1977), Die Metaphysik der Sitten, in: ders., Werkausgabe, Bd. 8, Frankfurt/M.

Kuenzle, D. u. M. Schefczyk (2009), John Stuart Mill. Zur Einführung, Hamburg.

Marx, K. u. F. Engels (1846/1978), Die deutsche Ideologie. Kritik der neuesten deutschen Philosophie in ihren Repräsentanten Feuerbach, B. Bauer und Stirner, und des deutschen Sozialismus in seinen verschiedenen Propheten, in: Marx-Engels-Werke, Bd. 3, Berlin.

Mill, J. S. (1861/1969), Utilitarianism, , in: ders., Collected Works, Bd. 10, Toronto, 203-259.

Ders. (1865/1979), An Examination of Sir William Hamilton's Philosophy, in: ders., Collected Works, Bd. 9, Toronto.

Nida-Rümelin, J. (2001), Strukturelle Rationalität. Ein philosophischer Essay über praktische Vernunft, Stuttgart.

Sher, G. (1997), Beyond Neutrality. Perfectionism and Politics, Cambridge.

Siep, L. (2002), Selbstverwirklichung, Anerkennung und politische Existenz. Zur Aktualität der politischen Philosophie Hegels, in: R. Schmücker u. U. Steinvorth (Hg.), Gerechtigkeit und Politik, Deutsche Zeitschrift für Philosophie, Sonderband 3, 41-56.

Skorupski, J. (2006), Green and the Idealist Conception of a Person's Good, in: M. Dimova-Cookson u. W. J. Mander (Hg.), T. H. Green. Ethics, Metaphysics, and Political Philosophy, Oxford, 47-75.

Theunissen, M. (1982), Selbstverwirklichung und Allgemeinheit. Zur Kritik des gegenwärtigen Bewußtseins, Berlin.

Tugendhat, E. (1979), Selbstbewußtsein und Selbstbestimmung. Sprachanalytische Interpretationen, Frankfurt/M.

\begin{abstract}
The article distinguishes two models of self-realization. The independence model claims that selfrealization is compatible with leading a non-moral life, whereas the dependence model argues the converse. Hegel's influential version of the dependence model aims at showing why and how self-realization must be embedded in a complex structure of reasonable social relations. I argue that Hegel's dependence model abrogates the "Recht der Besonderheit, sich befriedigt zu finden" and is thus not convincing. What I call Hegel's "inofficial theory", however, concedes an infusible conflict between modernity and self-realization; philosophy has to reconcile the individual with the impossibility of being a "ganzer Mensch" in modern societies. After an interlude with Michael Theunissen's indpendence model, I turn to T. H. Green's theory of self-realization. Green provides a richer explanatory story than Hegel as to why other-regarding acts contribute to self-realization; however, this story leaves not enough conceptual room for interpersonal conflict and is vulnerable to similar objections to Hegel's account
\end{abstract}

